\title{
Existence of solutions of integral equations via fixed point theorems
}

\author{
Selma Gülyaz ${ }^{1}$ and İnci M Erhan²*
}

\section{${ }^{*}$ Correspondence:}

ierhan@atilim.edu.tr

${ }^{2}$ Department of Mathematics,

Atilim University, İncek, Ankara

06836, Turkey

Full list of author information is

available at the end of the article

\begin{abstract}
Existence and uniqueness of fixed points of a mapping defined on partially ordered $G$-metric spaces is discussed. The mapping satisfies contractive conditions based on certain classes of functions. The results are applied to the problems involving contractive conditions of integral type and to a particular type of initial value problems for the nonhomogeneous heat equation in one dimension. This work is a generalization of the results published recently in (Gordji et al. in Fixed Point Theory Appl. 2012:74, 2012, doi:10.1186/1687-1812-2012-74) to G-metric space.
\end{abstract}

MSC: $47 \mathrm{H} 10 ; 54 \mathrm{H} 25$

Keywords: fixed point theory; G-metric spaces; initial value problems

\section{Introduction and preliminaries}

One of the most common applications of the fixed point theory is the problem of existence and uniqueness of solutions of initial and boundary value problems for differential and integral equations. The number of studies dealing with such problems has increased considerably in the recent years. An important result in this direction has been reported by JJ Nieto and RR Lopez in 2005 [1]. They studied existence and uniqueness of fixed points on partially ordered metric spaces and applied their results to boundary value problems for ordinary differential equations. The research in this direction is advancing continuously and produces many interesting results; see [2-6].

In 2006, Mustafa and Sims [7] introduced the concept of a G-metric and G-metric space, which is a generalization of metric space. After this pioneering work, $G$-metric spaces and particularly fixed points of various maps on $G$-metric spaces have been intensively studied; see [8-25] and also [26-29].

In this work, we present some fixed point theorems on $G$-metric spaces and investigate the existence of solutions of an initial value problem for a partial differential equation, more precisely, a nonlinear one dimensional heat equation.

First, we briefly introduce some basic notions of $G$-metric and $G$-metric space [7].

Definition 1.1 Let $X$ be a nonempty set, $G: X \times X \times X \rightarrow[0, \infty)$ be a function satisfying the following conditions:

(G1) $G(x, y, z)=0$ if $x=y=z$,

(G2) $0<G(x, x, y)$ for all $x, y \in X$ with $x \neq y$,

(G3) $G(x, x, y) \leq G(x, y, z)$ for all $x, y, z \in X$ with $z \neq y$,

(G4) $G(x, y, z)=G(x, z, y)=G(y, z, x)=\cdots$ (symmetry in all variables), 
(G5) $G(x, y, z) \leq G(x, a, a)+G(a, y, z)$ for all $x, y, z, a \in X$ (rectangle inequality).

Then the function $G$ is called a $G$-metric on $X$ and the pair $(X, G)$ is called a $G$-metric space.

Note that conditions (G4) and (G5) imply that

$$
|G(x, y, y)-G(x, z, z)| \leq 2 G(y, z, z)
$$

for all $x, y, z \in X$.

Definition 1.2 (see [7]) Let $(X, G)$ be a $G$-metric space and let $\left\{x_{n}\right\}$ be a sequence in $X$.

1. A point $x \in X$ is said to be the limit of the sequence $\left\{x_{n}\right\}$ if

$$
\lim _{n, m \rightarrow \infty} G\left(x, x_{n}, x_{m}\right)=0
$$

and the sequence $\left\{x_{n}\right\}$ is said to be $G$-convergent to $x$.

2. A sequence $\left\{x_{n}\right\}$ is called a $G$-Cauchy sequence if for every $\varepsilon>0$, there is a positive integer $N$ such that $G\left(x_{n}, x_{m}, x_{l}\right)<\varepsilon$ for all $n, m, l \geq N$; that is, if $G\left(x_{n}, x_{m}, x_{l}\right) \rightarrow 0$ as $n, m, l \rightarrow \infty$.

3. $(X, G)$ is said to be $G$-complete (or a complete $G$-metric space) if every $G$-Cauchy sequence in $(X, G)$ is $G$-convergent in $X$.

Proposition 1.3 (see [7]) Let $(X, G)$ be a G-metric space, $\left\{x_{n}\right\}$ be a sequence in $X$ and $x \in X$. Then the following are equivalent:

1. $\left\{x_{n}\right\}$ is G-convergent to $x$,

2. $G\left(x_{n}, x_{n}, x\right) \rightarrow 0$, as $n \rightarrow \infty$,

3. $G\left(x_{n}, x, x\right) \rightarrow 0$, as $n \rightarrow \infty$,

4. $G\left(x_{n}, x_{m}, x\right) \rightarrow 0$, as $n, m \rightarrow \infty$.

Proposition 1.4 (see [7]) The following statements are equivalent on a G-metric space $(X, G)$ :

1. The sequence $\left\{x_{n}\right\}$ is G-Cauchy.

2. For every $\varepsilon>0$, there is $N \in \mathbb{N}$ such that $G\left(x_{n}, x_{m}, x_{m}\right)<\varepsilon$, for all $n, m \geq N$.

Definition 1.5 (see [7]) Let $(X, G)$ and $\left(X^{\prime}, G^{\prime}\right)$ be $G$-metric spaces. A function $f:(X, G) \rightarrow$ $\left(X^{\prime}, G^{\prime}\right)$ is said to be $G$-continuous at a point $a \in X$ if and only if for every $\varepsilon>0$, there exists $\delta>0$ such that $x, y \in X$ and $G(a, x, y)<\delta$ implies $G^{\prime}(f(a), f(x), f(y))<\varepsilon$. A function $f$ is $G$ continuous on $X$ if and only if it is $G$-continuous at all points in $X$.

Proposition 1.6 Let $(X, G)$ be a G-metric space. Then the function $G(x, y, z)$ is jointly continuous in all of its three variables.

Definition 1.7 A $G$-metric space $(X, G)$ is said to be symmetric if

$$
G(x, x, y)=G(x, y, y)
$$

holds for arbitrary $x, y \in X$. Otherwise, the space is called asymmetric. 
It is obvious that for every $G$-metric on the set $X$, the expression

$$
d_{G}(x, y)=G(x, x, y)+G(x, y, y)
$$

is a standard metric on $X$.

Note that on a symmetric $G$-metric space $d_{G}(x, y)=2 G(x, y, y)$, but on an asymmetric $G$-metric space, the inequality

$$
\frac{3}{2} G(x, y, y) \leq d_{G}(x, y) \leq 2 G(x, y, y)
$$

holds for all $x, y \in X$.

Some examples of $G$-metric spaces are presented below.

\section{Example 1.8}

(1) Let $(X, d)$ be a metric space. Define $G s$ by

$$
G s(x, y, z)=d(x, y)+d(y, z)+d(x, z)
$$

for all $x, y, z \in X$. Then clearly, $(X, G s)$ is a symmetric $G$-metric space. Note that if $X=\mathbb{R}^{2}$ and $d$ is the Euclidean metric on $X$, then Gs may be interpreted as the perimeter of the triangle with vertices $x, y, z$.

(2) Let $X=\{a, b\}$. Define

$$
\begin{aligned}
& G(a, a, a)=G(b, b, b)=0, \\
& G(a, a, b)=1, \quad G(a, b, b)=2,
\end{aligned}
$$

and extend $G$ to $X \times X \times X$ by using the symmetry in the variables. Then $(X, G)$ is an asymmetric $G$-metric space.

\section{The main results}

The attempts to generalize the contractive conditions on the maps resulted in definitions of various classes of functions. Altering distance functions defined in [30], weak $\psi$-contraction presented in [31] are some of these classes. In this study we employ contractive conditions based on the following classes of functions.

Let $\Psi$ denote the class of the functions $\psi:[0,+\infty) \rightarrow[0,+\infty)$ satisfying the following conditions:

1. $\psi$ is nondecreasing,

2. $\psi$ is sub-additive, that is, $\psi(s+t) \leq \psi(s)+\psi(t)$,

3. $\psi$ is continuous,

4. $\psi(t)=0 \Longleftrightarrow t=0$.

Let $S$ denote the class of the functions $\beta:[0,+\infty) \rightarrow[0,1]$ such that for any bounded sequence $\left\{t_{n}\right\}$ of positive real numbers, $\beta\left(t_{n}\right) \rightarrow 1 \Longrightarrow t_{n} \rightarrow 0$.

Before stating our main results, we give the following auxiliary lemma which is going to be needed in the sequel. 
Lemma 2.1 Let $(X, G)$ be a G-metric space and let $\left\{x_{n}\right\}$ be a sequence in $X$ such that the sequence $\left\{G\left(x_{n+1}, x_{n+1}, x_{n}\right)\right\}$ of nonnegative real numbers is decreasing and

$$
\lim _{n \rightarrow \infty} G\left(x_{n+1}, x_{n+1}, x_{n}\right)=0 .
$$

When the subsequence $\left\{x_{2 n}\right\}$ is not G-Cauchy, then there exist $\varepsilon>0$ and two sequences $\left\{m_{k}\right\}$ and $\left\{n_{k}\right\}$ of positive integers such that the sequences

$$
\begin{array}{ll}
G\left(x_{2 m_{k}}, x_{2 n_{k}}, x_{2 m_{k}+1}\right), & G\left(x_{2 m_{k}}, x_{2 n_{k}+1}, x_{2 m_{k}+1}\right), \\
G\left(x_{2 m_{k}-1}, x_{2 n_{k}}, x_{2 m_{k}}\right), & G\left(x_{2 m_{k}-1}, x_{2 n_{k}+1}, x_{2 m_{k}}\right)
\end{array}
$$

converge to $\varepsilon$ as $k \rightarrow \infty$.

Proof From the Proposition 1.4, if $\left\{x_{2 n}\right\}$ is not G-Cauchy, then there exist $\varepsilon>0$ and two sequences $\left\{m_{k}\right\}$ and $\left\{n_{k}\right\}$ of $\mathbb{N}$ satisfying $n_{k}>m_{k}>k$ for which

$$
G\left(x_{2 m_{k}}, x_{2 n_{k}}, x_{2 m_{k}+1}\right) \geq \varepsilon
$$

where $n_{k}$ is chosen as the smallest integer satisfying (3). In other words,

$$
G\left(x_{2 m_{k}}, x_{2 n_{k}-2}, x_{2 m_{k}+1}\right)<\varepsilon \text {. }
$$

By (3), (4), and using the symmetry (G4) and the rectangle inequality (G5), we easily derive

$$
\begin{aligned}
\varepsilon \leq & G\left(x_{2 m_{k}}, x_{2 n_{k}}, x_{2 m_{k}+1}\right)=G\left(x_{2 m_{k}}, x_{2 m_{k}+1}, x_{2 n_{k}}\right) \\
\leq & G\left(x_{2 m_{k}}, x_{2 m_{k}+1}, x_{2 n_{k}-2}\right)+G\left(x_{2 n_{k}-2}, x_{2 n_{k}-2}, x_{2 n_{k}-1}\right) \\
& +G\left(x_{2 n_{k}-1}, x_{2 n_{k}-1}, x_{2 n_{k}}\right) \\
< & \varepsilon+G\left(x_{2 n_{k}-2}, x_{2 n_{k}-2}, x_{2 n_{k}-1}\right)+G\left(x_{2 n_{k}-1}, x_{2 n_{k}-1}, x_{2 n_{k}}\right) .
\end{aligned}
$$

Taking the limit $k \rightarrow \infty$ in (5) and using (1), we obtain

$$
\lim _{n \rightarrow \infty} G\left(x_{2 m_{k}}, x_{2 n_{k}}, x_{2 m_{k}+1}\right)=\varepsilon
$$

In addition, from the inequalities

$$
G\left(x_{2 m_{k}}, x_{2 n_{k}}, x_{2 m_{k}+1}\right) \leq G\left(x_{2 m_{k}}, x_{2 m_{k}+1}, x_{2 n_{k}+1}\right)+G\left(x_{2 n_{k}+1}, x_{2 n_{k}+1}, x_{2 n_{k}}\right)
$$

and

$$
G\left(x_{2 m_{k}}, x_{2 m_{k}+1}, x_{2 n_{k}+1}\right) \leq G\left(x_{2 m_{k}}, x_{2 m_{k}+1}, x_{2 n_{k}}\right)+G\left(x_{2 n_{k}}, x_{2 n_{k}}, x_{2 n_{k}+1}\right)
$$

we deduce

$$
\lim _{n \rightarrow \infty} G\left(x_{2 m_{k}}, x_{2 n_{k}+1}, x_{2 m_{k}+1}\right)=\varepsilon
$$


upon taking the limit $k \rightarrow \infty$ and using (1) and (6). In a similar way it can be shown that the remaining two sequences in (2) also tend to $\varepsilon$.

We state next our first main theorem about the existence of fixed points on partially ordered G-metric spaces.

Theorem 2.2 (Existence theorem) Let $(X, \preceq)$ be a partially ordered set, $(X, G)$ be a $G$ complete metric space and $f: X \rightarrow X$ be a nondecreasing function. Suppose that there exist functions $\beta \in S$ and $\psi \in \Psi$ such that

$$
\psi\left(G\left(f x, f y, f^{2} x\right)\right) \leq \beta(\psi(G(x, y, f x))) \psi(G(x, y, f x))
$$

for all $x, y \in X$ with $x \preceq y$. Assume also that for any increasing sequence $\left\{x_{n}\right\}$ in $X$ converging to $x$,

$$
x_{n} \preceq x \text { for each } n \geq 0 .
$$

If there exists $x_{0} \in X$ such that $x_{0} \preceq f x_{0}$, then $f$ has a fixed point.

Proof By the assumption, there exists $x_{0} \in X$ such that $x_{0} \preceq f x_{0}$. We construct a sequence $\left\{x_{n}\right\}$ in the following way:

$$
f x_{n}=x_{n+1} \quad \text { for all } n \in \mathbb{N} \cup\{0\} .
$$

Since $f$ is nondecreasing, we have $f x_{n} \preceq f x_{n+1}$ for each $n \in \mathbb{N} \cup\{0\}$. Hence, $\left\{x_{n}\right\}$ is a nondecreasing sequence. If $x_{n_{0}}=x_{n_{0}+1}$ for some $n_{0} \in \mathbb{N} \cup\{0\}$, then $x_{n_{0}}$ is the fixed point of $f$. Assume that $x_{n} \neq x_{n+1}$ for all $n \in \mathbb{N} \cup\{0\}$. Then, by the definition of $\psi$, we have $\psi\left(G\left(f x_{n}, f x_{n+1}, f^{2} x_{n}\right)\right)>0$ for all $n \in \mathbb{N} \cup\{0\}$. Taking $x=x_{n}$ and $y=x_{n+1}$ in (7) we get

$$
\begin{aligned}
\psi\left(G\left(x_{n+1}, x_{n+2}, x_{n+2}\right)\right) & =\psi\left(G\left(f x_{n}, f x_{n+1}, f^{2} x_{n}\right)\right) \\
& \leq \beta\left(\psi\left(G\left(x_{n}, x_{n+1}, x_{n+1}\right)\right)\right) \psi\left(G\left(x_{n}, x_{n+1}, x_{n+1}\right)\right) \\
& \leq \psi\left(G\left(x_{n}, x_{n+1}, x_{n+1}\right)\right) .
\end{aligned}
$$

Thus, the sequence $\left\{\psi\left(G\left(x_{n}, x_{n+1}, x_{n+1}\right)\right)\right\}$ is nonincreasing and bounded below by 0 . Consequently, $\lim _{n \rightarrow \infty} \psi\left(G\left(x_{n}, x_{n+1}, x_{n+1}\right)\right)=L \geq 0$. We will show that $L=0$. Assume to the contrary that $L>0$. Due to (7), we have

$$
\frac{\psi\left(G\left(f x_{n}, f x_{n+1}, f^{2} x_{n}\right)\right)}{\psi\left(G\left(f x_{n-1}, f x_{n}, f^{2} x_{n-1}\right)\right)} \leq \beta\left(\psi\left(G\left(x_{n}, x_{n+1}, x_{n+1}\right)\right)\right) \leq 1
$$

for each $n \geq 1$, which yields

$$
\lim _{n \rightarrow \infty} \beta\left(\psi\left(G\left(x_{n}, x_{n+1}, x_{n+1}\right)\right)\right)=1 .
$$

However, since $\beta \in S$, we have $\lim _{n \rightarrow \infty} \psi\left(G\left(f x_{n}, f x_{n+1}, f^{2} x_{n}\right)\right)=0$ and hence, $L=0$. 
We show next that $\left\{x_{n}\right\}$ is a G-Cauchy sequence. Suppose that $\left\{x_{n}\right\}$ is not G-Cauchy. By Lemma 2.1, there exist $\varepsilon>0$ and two sequences $\left\{m_{k}\right\}$ and $\left\{n_{k}\right\}$ of positive integers such that the four sequences

$$
\begin{aligned}
& G\left(f x_{2 m_{k}}, f x_{2 n_{k}}, f^{2} x_{2 m_{k}}\right), \quad G\left(f x_{2 m_{k}}, f x_{2 n_{k}+1}, f^{2} x_{2 m_{k}}\right), \\
& G\left(f x_{2 m_{k}-1}, f x_{2 n_{k}}, f^{2} x_{2 m_{k}-1}\right),
\end{aligned}
$$

approach $\varepsilon$ as $k$ goes to infinity. Setting $x=x_{2 m_{k}}$ and $y=x_{2 n_{k+1}}$ in (7) and regarding (9), we get

$$
\begin{aligned}
\psi & \left(G\left(f x_{2 m_{k}}, f x_{2 n_{k}+1}, f^{2} x_{2 m_{k}}\right)\right) \\
& \leq \beta\left(\psi\left(G\left(x_{2 m_{k}}, x_{2 n_{k}+1}, f x_{2 m_{k}}\right)\right)\right) \psi\left(G\left(x_{2 m_{k}}, x_{2 n_{k}+1}, f x_{2 m_{k}}\right)\right) \\
& =\beta\left(\psi\left(G\left(f x_{2 m_{k}-1}, f x_{2 n_{k}}, f^{2} x_{2 m_{k}-1}\right)\right)\right) \psi\left(G\left(f x_{2 m_{k}-1}, f x_{2 n_{k}}, f^{2} x_{2 m_{k}-1}\right)\right) \\
& \leq \psi\left(G\left(f x_{2 m_{k}-1}, f x_{2 n_{k}}, f^{2} x_{2 m_{k}-1}\right)\right),
\end{aligned}
$$

and thus

$$
\frac{\psi\left(G\left(f x_{2 m_{k}}, f x_{2 n_{k}+1}, f^{2} x_{2 m_{k}}\right)\right)}{\psi\left(G\left(f x_{2 m_{k}-1}, f x_{2 n_{k}}, f^{2} x_{2 m_{k}-1}\right)\right)} \leq \beta\left(\psi\left(G\left(f x_{2 m_{k}-1}, f x_{2 n_{k}}, f^{2} x_{2 m_{k}-1}\right)\right)\right) \leq 1
$$

This inequality implies $\lim _{k \rightarrow \infty} \beta\left(\psi\left(G\left(f x_{2 m_{k}-1}, f x_{2 n_{k}}, f^{2} x_{2 m_{k}-1}\right)\right)\right)=1$. Since $\beta \in S$, we conclude that

$$
\lim _{k \rightarrow \infty} \psi\left(G\left(f x_{2 m_{k}-1}, f x_{2 n_{k}}, f^{2} x_{2 m_{k}-1}\right)\right)=0 .
$$

By the fact that $\psi$ is a continuous function, $\psi(\varepsilon)=0$ and hence $\varepsilon=0$, which contradicts the assumption $\varepsilon>0$. Therefore, $\left\{f x_{n}\right\}$ is a $G$-Cauchy sequence in $(X, G)$. Since $(X, G)$ is a complete $G$-metric space, there exists $z \in X$ such that

$$
\lim _{n \rightarrow \infty} f x_{n}=z=\lim _{n \rightarrow \infty} x_{n+1}
$$

Next, we show that $z$ is a fixed point of $f$. Substituting $x=x_{n+1}$ and $y=z$ in (7), by the virtue of (10), we get

$$
\begin{aligned}
\psi & \left(G\left(x_{n+2}, f z, x_{n+3}\right)\right) \\
& =\psi\left(G\left(f x_{n+1}, f z, f^{2} x_{n+1}\right)\right) \\
& \leq \beta\left(\psi\left(G\left(x_{n+1}, z, f x_{n+1}\right)\right)\right) \psi\left(G\left(x_{n+1}, z, f x_{n+1}\right)\right) \\
& \leq \psi\left(G\left(x_{n+1}, z, f x_{n+1}\right)\right)=\psi\left(G\left(x_{n+1}, z, x_{n+2}\right)\right)
\end{aligned}
$$

for each $n \geq 1$. Passing to the limit $n \rightarrow \infty$ in the above inequality and regarding (10) and the continuity of $\psi$, we end up with

$$
G(z, f z, z)=0,
$$


that is

$$
f z=z .
$$

This completes the proof of the theorem.

Next, we discuss the uniqueness conditions for the fixed point of the map in Theorem 2.2. A condition for the uniqueness can be stated as follows:

(i) Every pair of elements in $X$ has a lower bound or an upper bound.

On the other hand, it can be proved that condition (i) is equivalent to condition

(ii) For every $x, y \in X$, there exists $z \in X$ which is comparable to both $x$ and $y$.

Accordingly, we prove the following uniqueness theorem.

Theorem 2.3 (Uniqueness theorem) Let X satisfies condition (ii) and the hypotheses of Theorem 2.2 hold. If $\beta \in S$ is continuous, then the fixed point off is unique.

Proof Existence of a fixed point is provided by Theorem 2.2. Assume that $y$ and $z$ are two different fixed points of $f$. From condition (ii), there exists $x \in X$ which is comparable to $y$ and $z$. The monotonicity of $f$ implies that $f^{n}(x)$ is comparable to $f^{n}(y)=y$ and $f^{n}(z)=z$ for $n \geq 0$. Moreover, using the fact that $z$ is a fixed point of $f$ and condition (7) of Theorem 2.2 we get

$$
\begin{aligned}
\psi\left(G\left(z, z, f^{n}(x)\right)\right) & =\psi\left(G\left(f^{n}(z), f^{n}(z), f^{n}(x)\right)\right) \\
& =\psi\left(G\left(f\left(f^{n-1}(z)\right), f\left(f^{n-1}(z)\right), f^{2}\left(f^{n-2}(x)\right)\right)\right),
\end{aligned}
$$

and thus

$$
\begin{aligned}
& \psi\left(G\left(z, z, f^{n}(x)\right)\right) \\
& \quad \leq \beta\left(\psi\left(G\left(f^{n-1}(z), f^{n-1}(z), f\left(f^{n-2}(x)\right)\right)\right)\right) \psi\left(G\left(f^{n-1}(z), f^{n-1}(z), f\left(f^{n-2}(x)\right)\right)\right) \\
& \quad \leq \psi\left(G\left(f^{n-1}(z), f^{n-1}(z), f^{n-1}(x)\right)\right)=\psi\left(G\left(z, z, f^{n-1}(x)\right)\right) .
\end{aligned}
$$

Therefore, the sequence $\left\{\alpha_{n}\right\}$ defined by $\alpha_{n}=\psi\left(G\left(z, z, f^{n}(x)\right)\right)$ is nonnegative and nonincreasing and hence,

$$
\lim _{n \rightarrow \infty} \psi\left(G\left(z, z, f^{n}(x)\right)\right)=\alpha \geq 0
$$

To show that $\alpha=0$, we assume the contrary, that is, $\alpha>0$. Since $\beta$ is continuous, $\lim _{n \rightarrow \infty} \beta\left(\alpha_{n}\right)=\beta(\alpha)=\lambda \geq 0$. Letting $n \rightarrow \infty$ in (13), we get

$$
\alpha \leq \lambda \alpha \leq \alpha,
$$

which results in $\lambda=1$. Since $\beta \in S$, we deduce

$$
\alpha=\lim _{n \rightarrow \infty} \alpha_{n}=\lim _{n \rightarrow \infty} \psi\left(G\left(z, z, f^{n}(x)\right)\right)=0 .
$$


By similar arguments, we obtain

$$
\lim _{n \rightarrow \infty} \psi\left(G\left(y, y, f^{n}(x)\right)\right)=0
$$

Employing the rectangle inequality (G4), we have

$$
G(z, z, y) \leq G\left(z, z, f^{n}(x)\right)+G\left(f^{n}(x), f^{n}(x), y\right)
$$

Since the inequality $G(x, x, y) \leq 2 G(x, y, y)$ holds for any $x, y \in X$ in both symmetric and asymmetric $G$-metric spaces, we have

$$
G(z, z, y) \leq G\left(z, z, f^{n}(x)\right)+2 G\left(f^{n}(x), y, y\right)
$$

From the fact that $\psi$ is nondecreasing and sub-additive, we conclude

$$
\psi(G(z, z, y)) \leq \psi\left(G\left(z, z, f^{n}(x)\right)\right)+2 \psi\left(G\left(f^{n}(x), y, y\right)\right)
$$

Letting $n \rightarrow \infty$ in the above inequality we obtain $\psi(G(z, z, y))=0$, which implies $G(z, z, y)=0$ and hence, $z=y$. This completes the proof.

If in Theorem 2.2, we take $\psi$ as the identity function on $X$ we deduce the following particular result.

Corollary 2.4 Let $(X, \preceq)$ be a partially ordered set $(X, G)$ be a $G$-complete metric space. Let $f: X \rightarrow X$ be a nondecreasing map. Suppose that there exists $\beta \in S$ such that

$$
G\left(f x, f y, f^{2} x\right) \leq \beta(G(x, y, f x)) G(x, y, f x)
$$

holds for all $x, y \in X$ with $x \preceq y$. Assume that either $f$ is continuous or that $X$ satisfies the following condition: if an increasing sequence $x_{n}$ in $X$ converges to $x$, then $x_{n} \preceq x$ for each $n \geq 0$. If in addition, there exists $x_{0} \in X$ such that $x_{0} \preceq f x_{0}$ then $f$ has a fixed point.

Remark 2.5 In a recent paper by Karapınar and Samet [32] it has been proven that if $d$ is a metric on $X$ and $\psi \in \Psi$, then the function $d_{\psi}=\psi(d(x, y))$ is also a metric on $X$. In a similar way, it can be shown that the function $G_{\psi}=\psi(G(x, y, z))$, where $\psi \in \Psi$ is also a G-metric. Employing this definition, the contractive condition in Theorem 2.2 can be simplified considerably. More precisely, it becomes

$$
G_{\psi}\left(f x, f y, f^{2} x\right) \leq \beta\left(G_{\psi}(x, y, f x)\right) G_{\psi}(x, y, f x)
$$

for all $x, y \in X$ with $x \preceq y$.

As a common application of fixed point theorems one can give integral type contractive conditions. In many articles authors apply their results to maps which are defined by integrals $[33,34]$. In what follows, we apply our results to maps defined by Lebesgue integrals. Let $Y$ be a set of functions $\chi: \mathbb{R}^{+} \rightarrow \mathbb{R}^{+}$satisfying the following conditions:

(1) $\chi$ is Lebesgue integrable; 
(2) $\chi$ is summable on each compact of subset of $\mathbb{R}^{+}$;

(3) $\chi$ is sub-additive;

(4) $\int_{0}^{\epsilon} \chi(t) d t>0$ for each $\epsilon>0$.

A sub-additive integrable function is defined as follows:

Definition 2.6 The function $\chi: \mathbb{R}^{+} \rightarrow \mathbb{R}^{+}$is called a sub-additive integrable function if, for any $a, b \in \mathbb{R}^{+}$, we have

$$
\int_{0}^{a+b} \chi(t) d t \leq \int_{0}^{a} \chi(t) d t+\int_{0}^{b} \chi(t) d t
$$

For the class of functions in $Y$, we state the following fixed point theorem.

Theorem 2.7 Let $(X, \preceq)$ be a partially ordered set and let $(X, G)$ be a complete G-metric space. Let $f: X \rightarrow X$ be a nondecreasing function. Suppose that there exist functions $\beta \in S$ and $\psi \in \Psi$ such that for $\chi \in Y$

$$
\int_{0}^{\psi\left(G\left(f x, f y, f^{2} x\right)\right)} \chi(t) d t \leq \beta\left(\int_{0}^{\psi(G(x, y, f x))} \chi(t) d t\right) \int_{0}^{\psi(G(x, y, f x))} \chi(t) d t
$$

holds for all $x, y \in X$ with $x \preceq y$. Assume that either $f$ is continuous or $X$ satisfies the condition: if an increasing sequence $\left\{x_{n}\right\}$ converges to $x$, then $x_{n} \preceq x$ for each $n \geq 0$. If there exists $x_{0} \in X$ such that $x_{0} \preceq f x_{0}$ then $f$ has a fixed point.

Proof For $\chi \in Y$, define the function $\Lambda: \mathbb{R}^{+} \rightarrow \mathbb{R}^{+}$by $\Lambda(x)=\int_{0}^{x} \chi(t) d t$. Observe that $\Lambda \in \Psi$. The inequality (15) can be written as

$$
\Lambda\left(\psi\left(G\left(f x, f y, f^{2} x\right)\right)\right) \leq \beta(\Lambda(\psi(G(x, y, f x))))(\Lambda(\psi(G(x, y, f x)))) .
$$

Let $\Lambda \circ \psi=\psi_{1}$, where clearly $\psi_{1} \in \Psi$. Then we have

$$
\psi_{1}\left(G\left(f x, f y, f^{2} x\right)\right) \leq \beta\left(\psi_{1}(G(x, y, f x))\right) \psi_{1}(G(x, y, f x)) .
$$

Then the conditions of Theorem 2.2 are satisfied and thus $f$ has a fixed point, which completes the proof.

The particular case in which the function $\psi$ is the identity function on $X$ can be stated as a corollary.

Corollary 2.8 Let $(X, \preceq)$ be a partially ordered set and, $(X, G)$ be a complete $G$-metric space. Let $f: X \rightarrow X$ be a nondecreasing map. Suppose that there exists $\beta \in S$ such that for $\chi \in Y$ the inequality

$$
\int_{0}^{G\left(f x, f y, f^{2} x\right)} \chi(t) d t \leq \beta\left(\int_{0}^{G(x, y, f x)} \chi(t) d t\right) \int_{0}^{G(x, y, f x)} \chi(t) d t
$$

holds for all $x, y \in X$ with $x \preceq y$. Assume that if an increasing sequence $\left\{x_{n}\right\}$ in $X$ converges to $x$ then $x_{n} \preceq x$ for each $n \geq 0$. If there exists $x_{0} \in X$ such that $x_{0} \preceq f x_{0}$ then $f$ has a fixed point. 


\section{Application}

As an application of the existence and uniqueness Theorems 2.2 and 2.3, we consider the problem of existence and uniqueness of an initial value problem defined by a nonlinear heat equation in one dimension. Such an initial value problem is defined as follows:

$$
\begin{cases}u_{t}(x, t)=u_{x x}(x, t)+F\left(x, t, u, u_{x}\right), & -\infty<x<\infty, 0<t<T, \\ u(x, 0)=\varphi(x), & -\infty<x<\infty,\end{cases}
$$

where $\varphi$ is assumed to be continuously differentiable, $\varphi$ and $\varphi^{\prime}$ bounded, and $F\left(x, t, u, u_{x}\right)$ a continuous function.

Definition 3.1 A solution of the initial value problem (17) is any function $u=u(x, t)$ defined in $\mathbb{R} \times I$, where $I=(0, T]$, satisfying the equation and the condition in (17) and also the conditions:

(a) $u \in C(\mathbb{R} \times I)$,

(b) $u_{t}, u_{x}$ and $u_{x x} \in C(\mathbb{R} \times I)$,

(c) $u$ and $u_{x}$ are bounded in $\mathbb{R} \times I$.

Consider the space $\Omega$ defined as

$$
\Omega=\left\{v(x, t): v, v_{x} \in C(\mathbb{R} \times I) \text { and }\|v\|<\infty\right\},
$$

where the norm on this space is defined as

$$
\|v\|=\sup _{x \in \mathbb{R}, t \in I}|v(x, t)|+\sup _{x \in \mathbb{R}, t \in I}\left|v_{x}(x, t)\right| .
$$

The set $\Omega$ endowed with the norm $\|\cdot\|$ defined in (18) is a Banach space. Define a $G$-metric on $\Omega$ as follows:

$$
\begin{aligned}
G(u, v, w)= & \sup _{x \in \mathbb{R}, t \in I}|u(x, t)-v(x, t)|+\sup _{x \in \mathbb{R}, t \in I}\left|u_{x}(x, t)-v_{x}(x, t)\right| \\
& +\sup _{x \in \mathbb{R}, t \in I}|v(x, t)-w(x, t)|+\sup _{x \in \mathbb{R}, t \in I}\left|v_{x}(x, t)-w_{x}(x, t)\right| \\
& +\sup _{x \in \mathbb{R}, t \in I}|u(x, t)-w(x, t)|+\sup _{x \in \mathbb{R}, t \in I}\left|u_{x}(x, t)-w_{x}(x, t)\right| .
\end{aligned}
$$

Then $(\Omega, G)$ is a complete $G$-metric space. Define also a partial order $\preceq$ on $\Omega$ as

$$
u, v \in \Omega, \quad u \preceq v \quad \Longleftrightarrow \quad u(x, t) \leq v(x, t), \quad u_{x}(x, t) \leq v_{x}(x, t)
$$

for any $x \in \mathbb{R}$ and $t \in I$. It can easily be observed that $(\Omega, \preceq$ ) satisfies condition (i) of uniqueness, that is, every pair of elements in $\Omega$ has a lower bound or an upper bound. Indeed, for any $u, v \in \Omega, \max \{u, v\}$ and $\min \{u, v\}$ are the lower and upper bounds for $u$ and $v$, respectively. Let $\left\{v_{n}\right\} \subseteq \Omega$ be a monotone nondecreasing sequence which converges to $v$ in $\Omega$. Then, for any $x \in \mathbb{R}$ and $t \in I$, we have

$$
v_{1}(x, t) \leq v_{2}(x, t) \leq \cdots \leq v_{n}(x, t) \leq \cdots
$$


and

$$
v_{1 x}(x, t) \leq v_{2 x}(x, t) \leq \cdots \leq v_{n x}(x, t) \leq \cdots .
$$

Moreover, since the sequences $\left\{v_{n}(x, t)\right\}$ and $\left\{v_{n x}(x, t)\right\}$ of real numbers converge to $v(x, t)$ and $v_{x}(x, t)$, respectively, we have for all $x \in \mathbb{R}, t \in I$ and $n \geq 1$ the inequalities $v_{n}(x, t) \leq$ $v(x, t)$ and $v_{n x}(x, t) \leq v_{x}(x, t)$ hold. Therefore, $v_{n} \leq v$ for all $n \geq 1$ and hence, $(\Omega, \preceq)$ with the $G$-metric defined above satisfies condition (8).

We next define a lower solution for the initial value problem, which is needed in the existence-uniqueness proof.

Definition 3.2 A lower solution of the initial value problem (17) is a function $u \in \Omega$ such that

$$
\begin{cases}u_{t}(x, t) \leq u_{x x}(x, t)+F\left(x, t, u, u_{x}\right), & -\infty<x<\infty, 0<t<T, \\ u(x, 0) \leq \varphi(x), & -\infty<x<\infty,\end{cases}
$$

where the function $\varphi$ is continuously differentiable and both $\varphi$ and $\varphi^{\prime}$ are bounded, the set $\Omega$ is the set defined above and $F\left(x, t, u, u_{x}\right)$ is a continuous function.

We state the following existence-uniqueness theorem for the solution of the initial value problem (17).

Theorem 3.3 Consider the problem (17) and, assume that $F: \mathbb{R} \times I \times \mathbb{R} \times \mathbb{R} \rightarrow \mathbb{R}$ is a continuous function. Suppose that the following conditions hold:

(1) For any $c>0$, the function $F(x, t, s, p)$, where $|s|<c$ and $|p|<c$ is uniformly Hölder continuous in $x$ and $t$, for each compact subset of $\mathbb{R} \times I$.

(2) There exists a constant $c_{F} \leq \frac{1}{3}\left(T+2 \pi^{-\frac{1}{2}} T^{\frac{1}{2}}\right)^{-1}$ such that

$$
0 \leq F\left(x, t, s_{2}, p_{2}\right)-F\left(x, t, s_{1}, p_{1}\right) \leq c_{F} \ln \left(s_{2}-s_{1}+p_{2}-p_{1}+1\right),
$$

for all $\left(s_{1}, p_{1}\right)$ and $\left(s_{2}, p_{2}\right)$ in $\mathbb{R} \times \mathbb{R}$ with $s_{1} \leq s_{2}$ and $p_{1} \leq p_{2}$.

(3) $F$ is bounded for bounded s and $p$.

Then the existence of a lower solution for the initial value problem (17) provides the existence of the unique solution of the problem (17).

Proof Observe that the problem (17) is equivalent to the integral equation

$$
\begin{aligned}
u(x, t)= & \int_{-\infty}^{\infty} k(x-\xi, t) \varphi(\xi) d \xi \\
& +\int_{0}^{t} \int_{-\infty}^{\infty} k(x-\xi, t-\tau) F\left(\xi, \tau, u(\xi, \tau), u_{\xi}(\xi, \tau)\right) d \xi d \tau
\end{aligned}
$$

for all $x \in \mathbb{R}$ and $0<t \leq T$, where the function $k(x, t)$ is the Green's function of the problem defined as

$$
k(x, t)=\frac{1}{\sqrt{4 \pi t}} \exp \left\{\frac{-x^{2}}{4 t}\right\}
$$


for all $x \in \mathbb{R}$ and $0<t$. The initial value problem (17) has a unique solution if and only if the above integral equation has unique solution $u$ such that $u$ and $u_{x}$ are continuous and bounded for all $x \in \mathbb{R}$ and $0<t \leq T$. Define a mapping $f: \Omega \rightarrow \Omega$ by

$$
\begin{aligned}
(f u)(x, t)= & \int_{-\infty}^{\infty} k(x-\xi, t) \varphi(\xi) d \xi \\
& +\int_{0}^{t} \int_{-\infty}^{\infty} k(x-\xi, t-\tau) F\left(\xi, \tau, u(\xi, \tau), u_{\xi}(\xi, \tau)\right) d \xi d \tau
\end{aligned}
$$

for all $x \in \mathbb{R}$ and $t \in I$. Clearly, the fixed point $u \in \Omega$ of $f$ is a solution of the problem (17). We will show that the conditions of Theorems 2.2 and 2.3 are satisfied. Note that the mapping $f$ is nondecreasing since, by condition (2) in the statement of the theorem, for $u \geq v$, that is, $u \geq v$ and $u_{x} \geq v_{x}$, we have

$$
F\left(x, t, u(x, t), u_{x}(x, t)\right) \geq F\left(x, t, v(x, t), v_{x}(x, t)\right) .
$$

Since $k(x, t)>0$ for all $(x, t) \in \mathbb{R} \times I$,

$$
\begin{aligned}
(f u)(x, t)= & \int_{-\infty}^{\infty} k(x-\xi, t) \varphi(\xi) d \xi \\
& +\int_{0}^{t} \int_{-\infty}^{\infty} k(x-\xi, t-\tau) F\left(\xi, \tau, u(\xi, \tau), u_{\xi}(\xi, \tau)\right) d \xi d \tau \\
\geq & \int_{-\infty}^{\infty} k(x-\xi, t) \varphi(\xi) d \xi \\
& +\int_{0}^{t} \int_{-\infty}^{\infty} k(x-\xi, t-\tau) F\left(\xi, \tau, v(\xi, \tau), v_{\xi}(\xi, \tau)\right) d \xi d \tau \\
= & (f v)(x, t)
\end{aligned}
$$

for all $x \in \mathbb{R}$ and $t \in I$. In addition, we have

$$
\begin{aligned}
& |(f u)(x, t)-(f v)(x, t)| \\
& \quad \leq \int_{0}^{t} \int_{-\infty}^{\infty} k(x-\xi, t-\tau)\left|F\left(\xi, \tau, u(\xi, \tau), u_{\xi}(\xi, \tau)\right)-F\left(\xi, \tau, v(\xi, \tau), v_{\xi}(\xi, \tau)\right)\right| d \xi d \tau \\
& \quad \leq \int_{0}^{t} \int_{-\infty}^{\infty} k(x-\xi, t-\tau) c_{F} \ln \left(u(\xi, \tau)-v(\xi, \tau)+u_{\xi}(\xi, \tau)-v_{\xi}(\xi, \tau)+1\right) d \xi d \tau \\
& \quad \leq c_{F} \ln (G(u, v, f u)+1) \int_{0}^{t} \int_{-\infty}^{\infty} k(x-\xi, t-\tau) d \xi d \tau \\
& \quad \leq c_{F} \ln (G(u, v, f u)+1) T
\end{aligned}
$$

where we have used the facts that

$$
\begin{aligned}
& \ln \left(u(\xi, \tau)-v(\xi, \tau)+u_{\xi}(\xi, \tau)-v_{\xi}(\xi, \tau)+1\right) \\
& \quad \leq \ln \left(2 \sup _{\xi \in \mathbb{R}, \tau \in I}|u(\xi, \tau)-v(\xi, \tau)|+2 \sup _{\xi \in \mathbb{R}, \tau \in I}\left|u_{\xi}(\xi, \tau)-v_{\xi}(\xi, \tau)\right|+1\right) \\
& \quad=\ln (G(u, v, f u)+1)
\end{aligned}
$$


and

$$
\int_{0}^{t} \int_{-\infty}^{\infty} k(x-\xi, t-\tau) d \xi d \tau=T .
$$

On the other hand, since $(\Omega, \preceq$ ) satisfies condition (i), it also satisfies condition (ii), as they are equivalent. Therefore, either $v$ and $f u$ are comparable or there exists $w \in \Omega$ which is comparable to both $v$ and $f u$. In either case, by means of similar calculations, it can be shown that

$$
\left|(f v)(x, t)-\left(f^{2} u\right)(x, t)\right| \leq c_{F} \ln (G(u, v, f u)+1) T
$$

and, similarly,

$$
\left|(f u)(x, t)-\left(f^{2} u\right)(x, t)\right| \leq c_{F} \ln (G(u, v, f u)+1) T
$$

for all $u \succeq v$. Moreover, by using differentiation under integral sign and employing again condition (2) of the theorem, we compute

$$
\begin{aligned}
\left|\frac{\partial f u}{\partial x}(x, t)-\frac{\partial f v}{\partial x}(x, t)\right| & \leq c_{F} \ln (G(u, v, f u)+1) \int_{0}^{t} \int_{-\infty}^{\infty}\left|\frac{\partial k}{\partial x}(x-\xi, t-\tau)\right| d \xi d \tau \\
& \leq c_{F} \ln (G(u, v, f u)+1) 2 \pi^{-\frac{1}{2}} T^{\frac{1}{2}}, \\
\left|\frac{\partial f v}{\partial x}(x, t)-\frac{\partial f^{2} u}{\partial x}(x, t)\right| & \leq c_{F} \ln (G(u, v, f u)+1) \int_{0}^{t} \int_{-\infty}^{\infty}\left|\frac{\partial k}{\partial x}(x-\xi, t-\tau)\right| d \xi d \tau \\
& \leq c_{F} \ln (G(u, v, f u)+1) 2 \pi^{-\frac{1}{2}} T^{\frac{1}{2}},
\end{aligned}
$$

and

$$
\begin{aligned}
\left|\frac{\partial f u}{\partial x}(x, t)-\frac{\partial f^{2} u}{\partial x}(x, t)\right| & \leq c_{F} \ln (G(u, v, f u)+1) \int_{0}^{t} \int_{-\infty}^{\infty}\left|\frac{\partial k}{\partial x}(x-\xi, t-\tau)\right| d \xi d \tau \\
& \leq c_{F} \ln (G(u, v, f u)+1) 2 \pi^{-\frac{1}{2}} T^{\frac{1}{2}}
\end{aligned}
$$

are satisfied. Combining (19), (22), and (23) with (24), (25), and (26), we deduce

$$
\begin{aligned}
G\left(f u, f v, f^{2} u\right) & \leq 3 c_{F}\left(T+2 \pi^{-\frac{1}{2}} T^{\frac{1}{2}}\right) \ln (G(u, v, f u)+1) \\
& \leq \ln (G(u, v, f u)+1)
\end{aligned}
$$

which implies

$$
\begin{aligned}
\ln \left(G\left(f u, f v, f^{2} u\right)+1\right) & \leq \ln (\ln (G(u, v, f u)+1)) \\
& =\frac{\ln (\ln (G(u, v, f u)+1))}{\ln (G(u, v, f u)+1)} \ln (G(u, v, f u)+1) .
\end{aligned}
$$

Define $\psi(x)=\ln (x+1)$ and $\beta(x)=\frac{\psi(x)}{x}$. Obviously, $\psi:[0, \infty) \rightarrow[0, \infty)$ is continuous, sub-additive, nondecreasing, and positive in $(0, \infty)$. Furthermore, $\psi(0)=0$ and also $\psi(x)<$ 
$x$ and hence, $\beta \in S$. Finally, let $\alpha(x, t)$ be a lower solution for (17). We show that $\alpha \leq f \alpha$. Upon integrating

$$
\begin{aligned}
& (\alpha(\xi, \tau) k(x-\xi, t-\tau))_{\tau}-\left(\alpha_{\xi}(\xi, \tau) k(x-\xi, t-\tau)\right)_{\xi}+\left(\alpha(\xi, \tau) k_{\xi}(x-\xi, t-\tau)\right)_{\xi} \\
& \quad \leq F\left(\xi, \tau, \alpha(\xi, \tau), \alpha_{\xi}(\xi, \tau)\right) k(x-\xi, t-\tau)
\end{aligned}
$$

over $-\infty<\xi<\infty$ and $0<\tau<t$, we obtain

$$
\begin{aligned}
\alpha(x, t) \leq & \int_{-\infty}^{\infty} k(x-\xi, t) \varphi(\xi) d \xi \\
& +\int_{0}^{t} \int_{-\infty}^{\infty} k(x-\xi, t-\tau) F\left(\xi, \tau, \alpha(\xi, \tau), \alpha_{\xi}(\xi, \tau)\right) d \xi d \tau \\
= & (f \alpha)(x, t)
\end{aligned}
$$

for all $x \in \mathbb{R}$ and $t \in(0, T]$. Therefore, by Theorems 2.2 and $2.3, f$ has a unique fixed point. This completes the proof.

\section{Competing interests}

The authors declare that they have no competing interests.

\section{Authors' contributions}

All authors contributed equally to this work. All authors read and approved the final manuscript.

\section{Author details}

${ }^{1}$ Department of Mathematics, Cumhuriyet University, Sivas, Turkey. ${ }^{2}$ Department of Mathematics, Atilim University, Incek, Ankara 06836, Turkey.

\section{Acknowledgements}

The authors are thankful to the referees for careful reading of the manuscript and the valuable comments and suggestions for the improvement of the paper.

Received: 28 November 2013 Accepted: 14 March 2014 Published: 03 Apr 2014

\section{References}

1. Nieto, JJ, Lopez, RR: Contractive mapping theorems in partially ordered sets and applications to ordinary differential equations. Order 22, 223-239 (2005)

2. Gordji, ME, Ramezani, M, Je Cho, Y, Pirbavafa, S: A generalization of Geraghty's theorem in partially ordered metric spaces and application to ordinary differential equations. Fixed Point Theory Appl. 2012, 74 (2012). doi:10.1186/1687-1812-2012-74

3. Agarwal, RP, El-Gebeily, MA, O'Regan, D: Generalized contractions in partially ordered metric spaces. Appl. Anal. 87, 1-8 (2008)

4. Bhaskar, TG, Lakshmikantham, V: Fixed point theory in partially ordered metric spaces and applications. Nonlinear Anal. 65, 1379-1393 (2006)

5. Lakshmikantham, V, Ćirić, LB: Coupled fixed point theorems for nonlinear contractions in partially ordered metric spaces. Nonlinear Anal. 70, 4341-4349 (2009)

6. Nashine, HK, Samet, B: Fixed point results for mappings satisfying $(\psi, \phi)$ weakly contractive condition in partially ordered metric spaces. Nonlinear Anal. 74, 2201-2209 (2011)

7. Mustafa, Z, Sims, B: A new approach to generalized metric spaces. J. Nonlinear Convex Anal. 7, $289-297$ (2006)

8. Agarwal, RP, Karapınar, E: Remarks on some coupled fixed point theorems in G-metric spaces. Fixed Point Theory Appl. 2013, 2 (2013). doi:10.1186/1687-1812-2013-2

9. Mustafa, Z, Sims, B: Fixed point theorems for contractive mappings in complete G-metric spaces. Fixed Point Theory Appl. 2009, 917175 (2009). doi:10.1155/2009/917175

10. Mustafa, Z, Obiedat, H, Awawdeh, F: Some fixed point theorem for mapping on complete G-metric spaces. Fixed Point Theory Appl. 2008, 189870 (2008). doi:10.1155/2008/189870

11. Mustafa, Z: A new structure for generalized metric spaces with applications to fixed point theory. Ph.D. thesis, The University of Newcastle, Australia (2005)

12. Mustafa, Z, Khandaqji, M, Shatanawi, W: Fixed point results on complete G-metric spaces. Studia Sci. Math. Hung. 48, 304-319 (2011)

13. Rao, KPR, Bhanu Lakshmi, K, Mustafa, Z: Fixed and related fixed point theorems for three maps in G-metric space. J. Adv. Stud. Topol. 3, 12-19 (2012) 
14. Mustafa, Z: Common fixed points of weakly compatible mappings in G-metric spaces. Appl. Math. Sci. 6, 4589-4600 (2012)

15. Mustafa, Z: Some new common fixed point theorems under strict contractive conditions in G-metric spaces. J. Appl. Math. 2012, Article ID 248937 (2012)

16. Mustafa, Z: Mixed $g$-monotone property and quadruple fixed point theorems in partially ordered G-metric spaces using $(\phi-\psi)$ contractions. Fixed Point Theory Appl. 2012(2012), 199 (2012)

17. Mustafa, Z, Shatanawi, W, Bataineh, M: Existence of fixed point results in G-metric spaces. Int. J. Math. Math. Sci. 2009, 283028 (2009). doi:10.1155/2009/283028

18. Aydi, H, Postolache, M, Shatanawi, W: Coupled fixed point results for $(\psi, \phi)$-weakly contractive mappings in ordered G-metric spaces. Comput. Math. Appl. 63, 298-309 (2012)

19. Aydi, H, Damjanović, B, Samet, B, Shatanawi, W: Coupled fixed point theorems for nonlinear contractions in partially ordered G-metric spaces. Math. Comput. Model. 54, 2443-2450 (2011)

20. Luong, NV, Thuan, NX: Coupled fixed point theorems in partially ordered G-metric spaces. Math. Comput. Model. 55, 1601-1609 (2012)

21. Aydi, H, Karapınar, E, Shatanawi, W: Tripled fixed point results in generalized metric spaces. J. Appl. Math. 2012, 314279 (2012). doi:10.1155/2012/314279

22. Aydi, H, Karapınar, E, Mustafa, Z: On common fixed points in G-metric spaces using (E.A) property. Comput. Math. Appl. 64, 1944-1956 (2012)

23. Tahat, N, Aydi, H, Karapınar, E, Shatanawi, W: Common fixed points for single-valued and multi-valued maps satisfying a generalized contraction in G-metric spaces. Fixed Point Theory Appl. 2012, 48 (2012). doi:10.1186/1687-1812-2012-48

24. Karapınar, E, Kaymakcalan, B, Tas, K: On coupled fixed point theorems on partially ordered G-metric spaces. J. Inequal. Appl. 2012, 200 (2012). doi:10.1186/1029-242X-2012-200

25. Ding, HS, Karapınar, E: A note on some coupled fixed point theorems on G-metric space. J. Inequal. Appl. 2012, 170 (2012). doi:10.1186/1029-242X-2012-170

26. Abbas, M, Sintunavarat, $W$, Kumam, P: Coupled fixed points of generalized contractive mappings on partially ordered G-metric spaces. Fixed Point Theory Appl. 2012, 31 (2012). doi:10.1186/1687-1812-2012-31

27. Sintunavarat, $W$, Kumam, P: Coupled coincidence and coupled common fixed points in partially ordered metric spaces. Thai J. Math. 10(3), 551-563 (2012)

28. Sintunavarat, W, Cho, YJ, Kumam, P: Coupled fixed points theorems for contraction mapping induced by cone ball-metric in partially ordered spaces. Fixed Point Theory Appl. 2012, 128 (2012). doi:10.1186/1687-1812-2012-128

29. Sintunavarat, W, Kumam, P: Coupled fixed points results for nonlinear integral equations. J. Egypt. Math. Soc. 21 266-272 (2013)

30. Khan, MS, Swaleh, M, Sessa, S: Fixed point theorems by altering distances between the points. Bull. Aust. Math. Soc. 30, 1-9 (1984)

31. Alber, YI, Guerre-Delabriere, S: Principles of weakly contractive maps in Hilbert spaces. In: New Results in Operator Theory and Its Applications, Operator Theory: Advances and Applications, pp. 7-22. Birkhäuser, Basel (1997)

32. Karapınar, E, Samet, B: A note on ' $\psi$-Geraghty type contractions'. Fixed Point Theory Appl. 2014, 26 (2014). doi:10.1186/1687-1812-2014-26

33. Karapınar, E, Erhan, IM, Yıldiz-Ulus, A: Cyclic contractions on G-metric spaces. Abstr. Appl. Anal. 2012, Article ID 182947 (2012). doi:10.1155/2012/182947

34. Jleli, M, Samet, B: Remarks on G-metric spaces and fixed point theorems. Fixed Point Theory Appl. 2012,210 (2012). doi:10.1186/1687-1812-2012-210

\section{Submit your manuscript to a SpringerOpen ${ }^{\ominus}$ journal and benefit from:}

- Convenient online submission

Rigorous peer review

- Immediate publication on acceptance

- Open access: articles freely available online

- High visibility within the field

- Retaining the copyright to your article 